03

\title{
Квазиоптическое уравнение в среде со слабой диссипацией
}

\author{
(C) Н.Н. Розанов ${ }^{1,2}$ \\ ${ }^{1}$ ФТИ им. А.Ф. Иофрфе, \\ 194021 Санкт-Петербург, Россия \\ ${ }^{2}$ Государственный оптический институт им. С.И. Вавилова, \\ 199053 Санкт-Петербург, Россия \\ e-mail: nnrosanov@mail.ru
}

Поступила в редакцию 02.02.2020 г.

В окончательной редакции 02.02.2020 г.

Принята к публикации 16.03.2020 г.

\begin{abstract}
Проведен анализ вида квазиоптического уравнения для импульса монохроматического излучения, распространяющегося в однородной или одномерно неоднородной линейной среде с диссипацией. Для слоя неоднородной среды получены эффективные параметры эквивалентной однородной среды.
\end{abstract}

Ключевые слова: квазиоптическое приближение, диссипативные эффекты.

DOI: $10.21883 /$ OS.2020.08.49710.69-20

Квазиоптическое приближение и уравнение были предложены около 75 лет тому назад [1,2] и затем прочно вошли не только в оригинальные статьи, но и в обзоры, учебники и монографии [3-8], охватывающие широкий круг оптических, радиофизических, акустических и других волновых процессов. Тем не менее эти вопросы до сих пор остаются актуальными ввиду большого разнообразия сред с существенно различающимися материальными соотношениями. Среди недавних публикаций отметим предложенный и использованный в $[9,10]$ вариант квазиоптического уравнения для плавно неоднородных сред с пространственной дисперсией.

Квазиоптическое приближение применимо к квазимонохроматическому и квазиплосковолновому излучению. Основная идея приближения применительно к оптике состоит в представлении полной напряженности электрического поля в виде произведения медленно меняющейся огибающей (в масштабах обратной частоты и длины волны опорной, несущей составляющей) на быстро меняющийся фазовый множитель [1-10]. Однако, как показано в [11], для слоя среды с хотя бы слабым поглощением такой подход не позволяет учесть то простое обстоятельство, что волны, распространяющиеся нормально к границам слоя, обладают меньшим суммарным поглощением, чем наклонно распространяющиеся волны (угловая селективность поглощения). Это обстоятельство приводит к тому, что обычная дифракция в средах с поглощением дополняется своеобразной диффузией, что вызывает своеобразные явления дихроизма (понимаемого здесь как зависимость поглощения от направления распространения волны) $[12,13]$. Для широкоапертурных лазерных систем, в которых присутствует диссипация в виде и поглощения, и усиления, указанная угловая селективность потерь представляется принципиальным фактором. Особенно ярко ее влияние проявляется для топологических структур света, обладающих сингулярностями волнового фронта [8,14-16].

Настоящая заметка является обобщением и дополнением предыдущей работы [11]. Новыми моментами служат анализ импульсного режима и учет продольной неоднородности среды. Результаты можно также рассматривать как обоснование постановки задачи о топологических трехмерных солитонах [14-16].

Начнем рассмотрение со случая распространения импульсов и пучков излучения, близких к монохроматической плоской волне, в однородной изотропной среде с диэлектрической проницаемостью $\varepsilon(\omega)=\varepsilon^{\prime}(\omega)+i \varepsilon^{\prime \prime}(\omega), \quad\left|\varepsilon^{\prime \prime}\right| \ll \varepsilon^{\prime}$. Здесь и далее один и два штриха обозначают вещественную и мнимую части; использовано спектральное представление, причем спектр частот $\omega$ со значимыми амплитудами поля сосредоточен в узкой области около несущей частоты $\omega_{0}$, а неравенство означает малость коэффициентов поглощения или усиления.

Согласно квазиоптическому приближению [1,2], представим полную электрическую напряженность электромагнитного поля $\tilde{\mathbf{E}}$ в виде

$$
\tilde{\mathbf{E}}=\operatorname{Re}\left\{\mathbf{E}(\mathbf{r}, t) \exp \left[i\left(k_{0} z-\omega_{0} t\right)\right]\right\}
$$

Здесь $\mathbf{r}=\{x, y, z\}-$ радиус-вектор, $x, y, z$ - декартовы координаты, $z$ - направление преимущественного распространения излучения, $t-$ время, $k_{0}=\frac{\omega_{0}}{c} \sqrt{\varepsilon\left(\omega_{0}\right)}-$ отвечающее несущей частоте (комплексное) волновое число и $\mathbf{E}$ - медленно меняющаяся во времени и пространстве (в масштабах $2 \pi / \omega_{0}$ и $2 \pi /\left|k_{0}\right|$ соответственно) огибающая. В этих приближениях из исходных уравнений Максвелла вытекает следующий вид квазиоптического уравнения, описывающего распространение излучения в линейной изотропной среде (второе при- 
ближение теории дисперсии, см., например, $[8])$ :

$$
2 i k_{0}\left(\frac{\partial \mathbf{E}_{\perp}}{\partial z}+\frac{1}{v_{\mathrm{gr}}} \frac{\partial \mathbf{E}_{\perp}}{\partial t}\right)+\Delta_{\perp} \mathbf{E}_{\perp}-D_{2} \frac{\partial^{2} \mathbf{E}_{\perp}}{\partial t^{2}}=0 .
$$

Здесь

$$
\begin{gathered}
k(\omega)=\frac{\omega}{c} \sqrt{\varepsilon(\omega)}, \quad \frac{1}{v_{\mathrm{gr}}}=\left(\frac{d k}{d \omega}\right)_{\omega=\omega_{0}}, \\
D_{2}=\left(k \frac{d^{2} k}{d \omega^{2}}\right)_{\omega=\omega_{0}} .
\end{gathered}
$$

Для прозрачной среды $\left(\varepsilon^{\prime \prime}=0\right)$ эти величины вещественны, $v_{\text {gr }}$ имеет смысл групповой скорости, а $D_{2}-$ коэффициент дисперсии второго порядка.

В первом приближении теории дисперсии уравнение (2) резко упрощается:

$$
\frac{\partial \mathbf{E}_{\perp}}{\partial z}+\frac{1}{v_{\mathrm{gr}}} \frac{\partial \mathbf{E}_{\perp}}{\partial t}=0 .
$$

Формально „уравнение переноса с комплексной скоростью“ (4) решается заменой переменной $t \rightarrow \tau=$ $=t-\frac{1}{v_{\mathrm{gr}}} z$ :

$$
\mathbf{E}_{\perp}=\mathbf{f}\left(t-\frac{1}{v_{\mathrm{gr}}} z\right), \quad \mathbf{f}=\mathbf{E}_{\perp}(z=0, t) .
$$

Выберем, например, гауссов вид огибающей импульса, падающего на среду с просветленной границей при $z=0$ :

$$
\mathbf{f}(z=0, t)=\mathbf{A} \exp \left(-\frac{t^{2}}{T^{2}}\right), \quad \omega_{0} T \gg 1 .
$$

Тогда

$$
\begin{aligned}
& \mathbf{E}_{\perp}(z, t)=\mathbf{A} \exp \left(-\left(t-\frac{1}{v_{\mathrm{gr}}} z\right)^{2} / T^{2}\right) \\
& =\mathbf{A} \exp \left(-\left(\left(t-u^{\prime} z\right)^{2}+\left(u^{\prime \prime} z\right)^{2}+2 i u^{\prime \prime} z\left(t-u^{\prime} z\right)\right)^{2} / T^{2}\right) .
\end{aligned}
$$

В (7) введено обозначение $u=\frac{1}{v_{\mathrm{gr}}}=u^{\prime}+i u^{\prime \prime}$. Как видно из (7), максимум профиля интенсивности, находившийся в начальный момент при $z=0$, достигается при $z>0$ в момент времени $t=u^{\prime} z$. Поэтому вещественную групповую скорость можно определить как $V_{\mathrm{gr}}=u^{\prime-1}=\left(d k^{\prime} / d \omega\right)_{\omega_{0}}^{-1}$. Также при фиксированном значении $z$ при сдвиге времени на $\delta t$ фаза излучения меняется на $\delta \Phi=2 T^{-2} u^{\prime \prime} z \delta t$. Это отвечает сдвигу средней частоты импульса $\Delta \omega=2 T^{-2} u^{\prime \prime} z$, пропорциональному $z$. Естественно, для справедливости квазиоптического приближения все поправочные члены должны оставаться много меньшими основных.

Обратимся теперь к полному квазиоптическому уравнению во втором порядке теории дисперсии (2). В „сопровождающей“ системе координат оно принимает вид

$$
2 i k_{0} \frac{\partial \mathbf{E}_{\perp}}{\partial z}+\Delta_{\perp} \mathbf{E}_{\perp}-D_{2} \frac{\partial^{2} \mathbf{E}_{\perp}}{\partial \tau^{2}}=0 .
$$

Для монохроматического излучения получаем уравнение параксиальной дифракции

$$
2 i k_{0} \frac{\partial \mathbf{E}_{\perp}}{\partial z}+\Delta_{\perp} \mathbf{E}_{\perp}=0 .
$$

Решение этого уравнения имеет обычный вид

$$
\begin{gathered}
\mathbf{E}_{\perp}\left(\mathbf{r}_{\perp}, z\right)=\frac{k_{0}}{2 \pi i z} \iint \mathbf{E}_{\perp}\left(\mathbf{r}_{\perp}^{\prime}, 0\right) \exp \left[\frac{i k_{0}}{2 z}\left(\mathbf{r}_{\perp}-\mathbf{r}_{\perp}^{\prime}\right)^{2}\right] d \mathbf{r}_{\perp}^{\prime}, \\
\mathbf{r}_{\perp}=(x, y) .
\end{gathered}
$$

Однако комплексность „волнового числа“ $k_{0}$ придает среде свойства дихроизма, рассмотренного более детально в [12,13]. Напомним, что учет „эффективной диффузии“ размывает дифракционные минимумы (интенсивность в них не достигает 0), а угловая расходимость излучения неограниченно убывает при увеличении длины пройденной трассы, что вызвано угловой селективностью поглощения. Если ввести „поперечную электрическую площадь“" импульса $\mathbf{S}_{\perp}=\iint \mathbf{E}_{\perp} d \mathbf{r}_{\perp}$, то для нее из (9) для ограниченных пучков следует правило сохранения $\frac{d \mathbf{S}_{\perp}}{d z}=0$.

Если отвлечься от поперечных эффектов и рассмотреть распространение импульса в плосковолновом приближении, то оно описывается уравнением

$$
2 i k_{0} \frac{\partial \mathbf{E}_{\perp}}{\partial z}-D_{2} \frac{\partial^{2} \mathbf{E}_{\perp}}{\partial \tau^{2}}=0 .
$$

Решение такого уравнения и при комплексном $k_{0} \mathrm{c}$ точностью до обозначений совпадает с решением для огибающей при поперечно одномерной дифракции (щелевые пучки). Для „электрической площади огибающей“ $\mathbf{S}_{e}=\iint \mathbf{E}_{\perp} d \tau$ из (11) следует $d \mathbf{S}_{e} / d z=0$. Подчеркнем, что эта величина отличается от электрической площади импульса, в которой фигурирует не огибающая, а полная напряженность поля $\mathbf{S}_{E}=\iint \tilde{\mathbf{E}} d t$; последняя сохраняется в любых средах - линейных и нелинейных, изотропных и анизотропных, однородных и неоднородных [17-19]. В теории самоиндуцированной прозрачности для среды с нелинейным и существенно нестационарным откликом электрическая площадь огибающей не сохраняется [20].

Более общим является случай плавно (по сравнению с длиной волны, отвечающей несущей частоте) неоднородной среды. Локально распространение описывается прежними соотношениями. В случае слоисто неоднородной среды, комплексная диэлектрическая проницаемость $\varepsilon$ которой зависит только от $z$, в режиме одностороннего распространения излучения вместо (1) следует записать

$$
\tilde{\mathbf{E}}=\operatorname{Re}\left\{\mathbf{E}\left(\mathbf{r}_{\perp}, z, t\right) \exp \left[i \int_{0}^{z} k_{0}(z) d z-i \omega_{0} t\right]\right\} .
$$


Спектральное разложение поля на входе среды (при $z=0)$ запишем в виде

$$
\begin{aligned}
\tilde{\mathbf{E}}\left(\mathbf{r}_{\perp}, z=0, t\right)= & \operatorname{Re}\left\{\exp \left(i \omega_{0} t\right) \sum_{\mathbf{k}_{\perp}, v} \mathbf{F}\left(\mathbf{k}_{\perp}, v\right)\right. \\
& \left.\times \exp \left(i \mathbf{k}_{\perp} \mathbf{r}_{\perp}-i v t\right)\right\} .
\end{aligned}
$$

Поперечный волновой вектор $\mathbf{k}_{\perp}$ и частотный сдвиг $v$ считаются вещественными. Мы можем разбить всю протяженность среды $L$ на $N$ слоев, в пределах которых изменение $\varepsilon$ несущественно. Это эквивалентно представлению зависимости $k_{0}(z)$ кусочно-постоянной (ступенчатой) функцией. Тогда поле на выходе (при $\left.z=L=\sum_{m=1}^{N} L_{m}\right)$

$$
\begin{aligned}
\tilde{\mathbf{E}}\left(\mathbf{r}_{\perp}, z=L, t\right)= & \operatorname{Re}\left[\exp \left(i \sum_{m=1}^{N} k_{0, m} L_{m}-i \omega_{0} t\right)\right. \\
& \left.\times E\left(\mathbf{r}_{\perp}, z=L, t\right)\right],
\end{aligned}
$$

где огибающая $E$ имеет вид

$$
\begin{aligned}
& E\left(\mathbf{r}_{\perp}, z=L, t\right)=\sum_{\mathbf{k}_{\perp}, v} F\left(\mathbf{k}_{\perp}, v\right) \exp \left(i \mathbf{k}_{\perp} \mathbf{r}_{\perp}-i v t\right) \\
& \times \exp \left(i v \sum_{m=1}^{N} \frac{L_{m}}{v_{\mathrm{gr}, m}}-i \mathbf{k}_{\perp}^{2} \sum_{m=1}^{N} \frac{L_{m}}{2 k_{0, m}}+i v^{2} \sum_{m=1}^{N} \frac{D_{2, m} L_{m}}{2 k_{0, m}}\right) .
\end{aligned}
$$

Для достаточно большого числа слоев $N$ суммирование по номерам слоев можно заменить интегрированием по $z$. Сопоставим теперь приведенное выражение с видом огибающей с той же несущей частотой в однородной среде, характеризуемой некоторыми эффективными параметрами $\bar{k}_{0}, \bar{v}_{\mathrm{gr}}$ и $\bar{D}_{2}$ :

$$
\begin{gathered}
E\left(\mathbf{r}_{\perp}, z, t\right)=\sum_{\mathbf{k}_{\perp}, v} F\left(\mathbf{k}_{\perp}, v\right) \exp \left(i \mathbf{k}_{\perp} \mathbf{r}_{\perp}-i v t\right) \\
\times \exp \left[i\left(\frac{v}{\bar{v}_{\mathrm{gr}}}-\frac{\mathbf{k}_{\perp}^{2}}{2 k_{0}}+\frac{\bar{D}_{2} v^{2}}{2 \bar{k}_{0}}\right) z\right] .
\end{gathered}
$$

Из сравнения (15) и (16) следует

$$
\begin{gathered}
\frac{1}{\bar{k}_{0}}=\frac{1}{L} \sum_{m=1}^{N} \frac{L_{m}}{k_{0, m}}, \quad \frac{1}{\bar{v}_{\mathrm{gr}}}=\frac{1}{L} \sum_{m=1}^{N} \frac{L_{m}}{v_{\mathrm{gr}, m}}, \\
\bar{D}_{2}=\frac{\bar{k}_{0}}{L} \sum_{m=1}^{N} \frac{D_{2, m} L_{m}}{k_{0, m}} .
\end{gathered}
$$

В интегральной форме

$$
\frac{1}{\bar{k}_{0}}=\frac{1}{L} \int_{0}^{L} \frac{d z}{k_{0}}, \quad \frac{1}{\bar{v}_{\mathrm{gr}}}=\frac{1}{L} \int_{0}^{L} \frac{d z}{v_{\mathrm{gr}}}, \quad \bar{D}_{2}=\frac{\bar{k}_{0}}{L} \int_{0}^{L} \frac{D_{2} d z}{k_{0}} .
$$

Тем самым введение эффективной однородной среды оказывается возможным. Подчеркнем, что определяемая таким образом величина „среднего волнового числа“ $\bar{k}_{0}$ для огибающей отличается от такового для полного поля в $(12) \tilde{k}_{0}=(1 / L) \int_{0}^{L} k_{0}(z) d z$. Напомним, однако, что в широкоапертурных системах со слоями с лазерным усилением возможно развитие усиленного спонтанного излучения в преимущественно поперечных направлениях [21]. Вызванные поглощением поправки должны превосходить поправки более высокого порядка квазиоптического приближения. Для пучка с характерным масштабом поперечных неоднородностей $w$ это ведет к требованию $\left|n^{\prime \prime} / n^{\prime}\right|>\left(k^{\prime} w\right)^{-2}[11]$, а для импульсов аналогичное требование заключается в превышении этими поправками тех поправок, которые вызваны пренебрежением высшими порядками теории дисперсии.

Обобщение на случай оптически нелинейных сред требует конкретизации вида нелинейности. При этом в общем случае интенсивность излучения отличается от квадрата модуля огибающей множителем $\exp \left(2 \tilde{k}_{0}^{\prime \prime} z\right)$. При характерном для установившихся структур условии $\tilde{k}_{0}^{\prime \prime}=0$ (баланс насыщенных поглощения и усиления) этот фактор, по-видимому, не имеет принципиального значения. При выполнении этого условия среда становится эффективно прозрачной для волн, распространяющихся параллельно оси $z$, тогда как для наклонно распространяющихся волн поглощение преобладает над усилением (эффективный коэффициент диффузии отличен от 0 и положителен). Это обстоятельство подтверждает правомерность введения эффективного коэффициента диффузии в задачах о лазерных солитонах $[8,14-16]$.

\section{Финансирование работы}

Данное исследование поддержано грантом РНФ 18-1200075 .

\section{Конфоликт интересов}

Автор заявляет, что у него нет конфликта интересов.

\section{Список литературы}

[1] Леонтович М.А. // Изв. АН СССР, сер. физ. 1944. Т. 8. C. $16-22$.

[2] Леонтович М.А., Фок В.А. // ЖЭТФ. 1946. Т. 16. № 7. C. 557-573.

[3] Фок B.A. Проблемы дифракции и распространения электромагнитных волн. М.: Советское радио, 1970.

[4] Малюжинец, Г.Д. // УФН. 1959. Т. 69. № 2. С. 321-334.

[5] Вайнштейн Л.А. Открытые резонаторы и открытые волноводы. М.: Советское радио, 1966.

[6] Виноградова М.Б., Руденко О.В., Сухоруков А.П. Теория волн. М.: Наука, 1979.

[7] Власов С.Н., Таланов В.И. Самофокусировка волн. Нижний Новгород: ИПФ РАН, 1997. 
[8] Розанов Н.Н. Диссипативные оптические солитоны. М.: Физматлит, 2011.

[9] Балакин А.А., Господчиков Е.Д., Шалашов А.Г. // Письма в ЖЭТФ. 2016. Т. 104. № 10. С. 701-707.

[10] Шалашов А.Г., Балакин А.А., Хусаинов Т.А., Господчиков Е.Д., Соломахин А.Л. // ЖЭТФ. 2017. Т. 151. № 2. C. 379-395.

[11] Розанов Н.Н. // Опт. и спектр. 2019. Т. 127. В. 2. C. 283-285; Rosanov N.N. // Opt. Spectrosc. 2019. V. 127. N 2. P. $285-287$.

[12] Розанов Н.Н. // Опт. и спектр. 2001. Т. 90. № 1. C. 136-138; Rozanov N.N. // Opt. Spectrosc. 2001. V. 90. N 1. P. $121-123$

[13] Розанов Н.Н. // Опт. и спектр. 2001. Т. 90. № 2. C. 311-314; Rozanov N.N. // Opt. Spectrosc. 2001. V. 90. N 2. P. $26-268$.

[14] Veretenov N.A., Rosanov N.N., Fedorov S.V. // Phys. Rev. Lett. 2016. V. 117. N 18. P. 183901.

[15] Veretenov N.A., Fedorov S.V., Rosanov N.N. // Phys. Rev. Lett. 2017. V. 119. N 26. P. 263901.

[16] Fedorov S.V., Veretenov N.A., Rosanov N.N. // Phys. Rev. Lett. 2019. V. 122. N 2. P. 023903.

[17] Розанов Н.Н. // Опт. и спектр. 2009. Т. 105. № 5. C. 799-803; Rosanov N.N. // Opt. Spectrosc. 2009. V. 107. N 5. P. $721-725$.

[18] Rosanov N.N., Kozlov V.V., Wabnitz S. // Phys. Rev. A. 2010. V. 81. N 4. P. 043815.

[19] Розанов Н.Н., Архипов Р.М., Архипов М.В. // УФН. 2018. T. 188. № 12. C. 1347-1353; Rosanov N.N., Arkhipov R.M., Arkhipov M.V. // Physics-Uspekhi. 2018. V. 61. N 12. P. $1227-1233$.

[20] McCall S.L., Hahn E.L. // Phys. Rev. 1969. V. 83. N 2. P. 457-485.

[21] Розанов Н.Н. // УФН. 2017. Т. 187. № 8. С. 879-882; Rosanov N.N. // Physics-Uspekhi. 2017. V. 60. N 8. P. $818-821$. 